\title{
Arthroscopic Remnant-Preserving Anterior Talofibular Ligament Reconstruction does not Improve the Short-term Function of Ankle
}

Shi-Ming Feng ( $\square$ fengshiming_04@163.com )

Xuzhou Central Hospital https://orcid.org/0000-0002-0815-2426

Nicola Maffulli

Universita degli Studi di Salerno Dipartimento di Medicina e Chirurgia

Francesco Oliva

Universita degli Studi di Salerno Dipartimento di Medicina e Chirurgia

Qing-Qing Sun

xuzhou central hospital

Ai-Guo Wang

Xuzhou Central Hospital

Research article

Keywords: Anterior talofibular ligament, Chronic lateral ankle instability, Ligament reconstruction, Remnant-preserving

Posted Date: January 8th, 2020

DOI: https://doi.org/10.21203/rs.2.20401/v1

License: (c) (1) This work is licensed under a Creative Commons Attribution 4.0 International License. Read Full License 


\section{Abstract}

Background Anterior talofibular ligament (ATFL) reconstruction is a valid treatment of chronic lateral ankle instability (CLAl). The purpose of this study was to evaluate the significance of preserving and not preserving remnant in ATFL reconstruction, as well as the clinical efficacy.

Methods From January 2015 to July 2017, fifty-three admitted remnant-preserving CLAl patients with ATFL injury were prospectively randomized as remnant-preserving group (preservation of ATFL) and nonpreserving group (no preservation of ATFL). All patients received anatomic reconstruction of ATFL using the semitendinosus tendon autograft. The Visual Analogue Scale (VAS) score, the American Orthopedic Foot and Ankle Society (AOFAS) score, Karlsson Ankle Functional Score (KAFS), Anterior Talar Translation (ATT), and ankle proprioceptive recovery in both groups were compared.

Results All patients were followed up for at least 2 years, and difference in the follow-up time between two groups was not statistically significant. Differences in VAS, AOFAS, KAFS, ATT and ankle proprioceptive recovery between two groups were not statistically significant.

Conclusions Compared with non-remnant-preserving surgery, there are no short-term follow-up differences in postoperative ankle function, stability and proprioceptive recovery when semitendinosus tendon autograft is used to treat CLAl through remnant-preserving ATFL reconstruction.

Level of Evidence: Level III, a prospective comparative study.

Trial registration: Xuzhou Central Hospital, ZXYY-2015090. Registered 21 November 2014

\section{Background}

The incidence of sport injury shows an increasing trend year by year with the emergence of sport craze and the deeper popular concept of sports promoting health. Ankle injury is the most common injury type among all sport injuries, while inversion injury is the most frequently seen in ankle injury [1]. Lateral ankle ligament complex is the most susceptible ligament structure to injury in ankle sport injury, especially for anterior talofibular ligament (ATFL). After ATFL injury, most patients can obtain satisfactory outcomes through conservative treatment and rehabilitation functional exercise [2-4]. Conservative treatment and rehabilitation functional exercise are effective in most of patients with an ankle sprain. However, surgical treatment is required for patients with the ankle instability of over 6 months or repeated ankle sprain [5]. Meanwhile, for patients with unrepairable ATFL injury or high exercise requirements (such as athletes), ligament reconstruction is an important surgical technique for treating ATFL injury $[6,7]$.

In ATFL reconstruction, how to treat the preserved ATFL remnant is a critical issue faced by the sports medicine physician in surgery. It remains unclear about whether remnant-preserving surgery is required in ATFL reconstruction, just like the remnant-preserving surgery in anterior cruciate ligament $(A C L)$ reconstruction [8]. To our knowledge, there is no comparative literature report or suggestion on whether 
remnant-preserving ATFL reconstruction is required, or whether remnant-preserving ATFL reconstruction has superior effect to non-preserving.

In this study, we explored and compared the clinical effects between remnant-preserving and nonpreserving ATFL reconstruction in terms of ankle function, stability and proprioceptive recovery.

\section{Methods}

Inclusion criteria: (1) patients who were diagnosed with chronic lateral ankle instability (CLAl) and received standard conservative treatment for over 6 months, with no improved symptom; (2) patients with unilateral ankle ATFL injury (non-avulsed fracture type) but no calcaneofibular ligament injury (confirmed under arthroscopy); (3) The fibular side of ATFL remnant was integral, as verified under arthroscope, and the ATFL could not be repaired directly; (4) patients who were willing or required to perform all arthroscopic ATFL reconstruction, and the transplanted tendon was the autologous semitendinosus tendon; (5) patients who received ankle ligament surgery for the first time, with no previous history of ankle injury or surgery; (6) patients with complete surgical and follow-up data, and were followed up for at least 24 months clinically.

Exclusion criteria: (1) patients combined with foot and ankle deformity, abnormal line of force, fracture, ankylosis and other ligament injuries; (2) those combined with central and peripheral neuromuscular atrophic disease or ligament laxity; (3) patients with ankle osteoarthritis or osteochondral injury that required osteochondral transplantation; (4) those with severe underlying disease and could not tolerate the surgery.

From January 2015 to July 2017, a total of 53 CLAl patients undergoing ATFL reconstruction were enrolled into the current study according to the above inclusion and exclusion criteria. The institutional review boards of our hospital approved our study protocol. All cases provided the informed consent as well as consents for the Health Insurance Portability and Accountabilty Act to participate in this study. All operations were completed by the same surgeon.

The above patients were randomized as remnant-preserving group (preservation of the ATFL remnant, $n=25$ ) and non-preserving group (no preservation of the ATFL remnant, $n=28$ ) according to the random number table method. There was no statistical difference in the general preoperative data between two groups; in addition, differences in Visual Analogue Scale (VAS) score, the American Orthopedic Foot and Ankle Society (AOFAS) score, Karlsson Ankle Functional Score (KAFS) [9], and Anterior Talar Translation (ATT) [10] between two groups were not statistically significant. (Table 1)

\section{Surgical technique}

The patient was in supine position, and a 7-cm cushion was placed in the affected hip after successful anesthesia, so that the affected leg was in the neutral position of ankle under natural anesthesia status. Then, the affected leg was placed in the distal edge of the operating table for convenient operation 
technique, the pressure pneumatic tourniquet was put in the middle segment of thigh, and the pneumatic pressure was set at $60 \mathrm{kPa}$ after blood evacuation.

First of all, the ipsilateral semitendinosus tendon of patient about $6-8 \mathrm{~cm}$ in length was excised, the soft tissue and muscular tissue on the tendon were completely cleaned, and only the tendinous tissue was preserved. Subsequently, the tendon was subjected to $10 \mathrm{~min}$ of pre-load at 150 pounds. A button plate (Smith \& Nephew, USA) that was $15 \mathrm{~mm}$ in length was selected, the tendon was penetrated into the button plate at equivalent length, and the both sides of tendon were then sutured.

The ankle anterior-lateral and anterior-medial standard approaches were adopted to explore and treat the lesion within the ankle joint, and the scars as well as inflammatory hypertrophic tissues were also cleared. Afterwards, the anterior subtalar approach was constructed to explore the ATFL, and the Footprint region on the fibula was also exposed. For the remnant-preserving group, the remnant on the ATFL fibula was preserved (Fig 1a); for the non-remnant-preserving group, the ATFL tendinous tissue was completely cleaned. Then, a guide pin was inserted into the Footprint region center of fibular from the anterior-inferior to the posterior-superior direction through this channel, which formed a $30^{\circ}$ angle with the fibular longitudinal axis (Fig 1b). Later, the $4.5 \mathrm{~mm}$ hollow drill was used to drill a bone tunnel, which should be long enough to penetrate the fibula (Fig 1c/1d). Then, the Footprint region on the talus was explored and cleaned through the ankle anterior-lateral approach and the anterior subtalar approach, a guide pin was inserted through the anterior subtalar approach, and the $6.0 \mathrm{~mm}$ hollow drill was utilized for drilling to form a bone tunnel that was $20 \mathrm{~mm}$ in depth.

The tendon graft was put into the lateral ankle through the anterior-lateral ankle approach (Fig 2a), later, the guide pin with a wire was used to penetrate the button plate into the fibular tunnel (Fig $2 \mathrm{~b}$ ), and then the other terminal of tendon graft was pulled back to better confirm the button plate fixing effect (Fig 2c). Afterwards, under the neutral position of ankle joint, the grafted tendon was strained, and a $6.0 \mathrm{~mm}$ interface screw (Smith \& Nephew, USA) was used to fix the distal end of grafted tendon inside the talar tunnel (Fig 2d and Fig 3).

\section{Postoperative management}

The short-legged bracket was used to fix the ankle joint for 2 weeks at mild dorsiflexion and eversion position. On the second day after surgery, the patient was advised to conduct early non-weight-bearing functional exercises or isometric exercises of lower limb muscles. Two weeks later, weight-bearing functional exercises were carried out in the presence of ankle Aircast ${ }^{\mathrm{TM}}$ (DJO, Vista, CA, USA), and physical activities were encouraged after removing the Aircast at 6 weeks later.

\section{Postoperative follow-up and observational indexes}

The patient wound healing and ankle stability were followed up after surgery; besides, the VAS, AOFAS, KAFS and ATT scores were adopted to assess the ankle function. The ankle proprioception was assessed 
using the Active Joint Position Sense (AJPS) [11]. All measurements were completed by the same rehabilitation physician who did not participate in surgery and was blind to grouping results.

\section{Statistical analysis}

The SPSS 19.0 statistical software was used for analysis. The measurement data, such as VAS, AOFAS, KAFS, ATT, and AJPS scores, in each group before and after surgery, as well as between two groups after surgery, were compared and analyzed using t test (symmetric distribution) or Mann-Whitney test (asymmetric distribution). Pearson chi-square test was adopted to compare the enumeration data, like age and sex. A difference of $P<0.05$ was deemed as statistically significant.

\section{Results}

All operations in 53 patients were successful, and all postoperative wounds achieved one-stage healing, with no wound complication or implant reaction, no nervous or vascular injury complication, the anterior drawer tests were all negative, and no semitendinosus tendon donor site-related complication was observed. Differences in operation time, length of hospital stay, and follow-up time between two groups were not statistically significant. In the last follow-up, the average VAS scores in remnant-preserving and non-remnant-preserving groups were 1.22 points (range, $0-2$ points) and 1.14 points (range, 0-2 points), respectively, and the difference was of no statistical significance. However, the last scores in both groups were markedly lower than those before surgery. The AOFAS scores in both groups were remarkably improved compared with those before surgery, and difference between two groups was not statistically significant. The KAFS score in remnant-preserving group was 88.01 points (range, 80-95 points), while that in non-remnant-preserving group was 87.15 points (range, $80-95$ points), and difference between two groups was not statistically significant. The ATT in remnant-preserving group was $2.87 \mathrm{~mm}$ (range, 2-4 $\mathrm{mm}$ ), while that in non-remnant-preserving group was $2.90 \mathrm{~mm}$ (range, 2-4 mm), and the results in both groups were evidently reduced compared with those before surgery, but the difference was not statistically significant. The AJPS in remnant-preserving group and non-remnant-preserving group was no statistical difference. (Table 2)

\section{Discussion}

The lateral complex ankle ligaments are constituted by ATFL, calcaneofibular ligament and posterior talofibular ligament, which is the important structure to maintain the ankle stability. The anatomical structure of narrow in the front and wide in the back of talus renders the ankle susceptible to varus sprain, while ATFL has the maximal tension when the ankle is at the varus plantarflexion position. Therefore, ATFL injury is the most commonly seen in clinic, which will result in CLAl if it is inappropriately treated $[12,13]$. CLAl accelerates ankle injury and degeneration, which then results in abnormal line of force and arthritis. CLAl can be classified as functional instability and mechanical instability. Of them, mechanical instability is the true ankle instability resulting from injury of the lateral ankle ligament complex, which requires repair or reconstruction of lateral ankle ligament $[14,15]$. The symptomatic instability is induced 
by proprioceptive dysfunction after ankle injury, without injury or rupture of the lateral ankle ligament complex [16]. Thus, the integrity of lateral ankle ligament and the proprioceptive function of ankle are two vital aspects affecting the ankle stability $[17,18]$.

ATFL reconstruction is an important surgical treatment to recover the lateral ankle ligament structure and ankle stability. ATFL reconstruction can be divided into open surgery and arthroscopic surgery. Of them, open surgery can achieve good ankle joint function and long-term follow-up outcomes. However, open surgery is also associated with the drawbacks of large wound, slow postoperative recovery, susceptibility to early ankle and subtalar ankylosis, and later ligament laxity as well as lateral ankle instability recurrence, which thus can not attain satisfactory long-term effects, and is rarely used in clinic at present $[19,20]$. By contrast, arthroscopic reconstruction is linked with the advantages of less local tissue separation, and being able to observe and treat the ATFL as well as intra-articular lesion, which is extensively applied in recent years and attains superb clinical effects [21,22]. The allogeneic tendon or autologous tendon can be used as the ATFL reconstruction tendon material; typically, autologous tendon transplantation, which has the advantages of no postoperative rejection reaction, convenient sampling, and little donor site complication, is the major graft material in ATFL reconstruction [23]. Autologous semitendinosus tendon transplantation is one of the most widely applied autologous tendons [24]. From the tendon transplantation effects of our patients, autologous semitendinosus tendon graft for reconstructing ATFL achieved satisfying short-term clinical effects, with no complications in donor site and no graft rejection after surgery.

Proprioceptive functional reconstruction after chronic ankle injury has always been the important issue that attracts the attention from both sports medicine physicians and rehabilitation physicians $[17,25]$. The mechanical sensor of ankle is located in the lateral ankle ligament, articular capsule and extensor retinaculum, and injury in these structures results in proprioceptive dysfunction in patient, which may also result in symptomatic ankle instability [26]. Therefore, how to maximally protect these tissues during ankle ligament reconstruction has great impact on the proprioceptive functional recovery of patient. In ATFL reconstruction, we not only attach importance to the recovery of ATFL anatomical structure, but also pay attention to the protection of ankle proprioception, so as to facilitate the rapider recovery of postoperative ankle function.

ATFL has similar structure to ACL, and has the proprioceptor. Similar to the remnant-preserving ACL reconstruction, remnant preservation may accelerate the revascularization and synovial membrane covering of the graft ligament structure [27]. As a result, some scholars advocate the ATFL-preserving remnant ligament reconstruction. Dong et al. reported the surgical effects of 20 cases receiving remnantpreserving lateral ankle ligament reconstruction, and suggested that, the ATFL-preserving remnant better recovered the function after ankle ligament reconstruction, and emphasized the importance of remnantpreserving surgery and favorable clinical follow-up results [28]. However, Wang et al. performed ATFL reconstruction to treat 19 CLAl cases, no ATFL ligament remnant was preserved introperatively, and patients were followed up for an average of 30 months after surgery; their results suggested that, the AOFAS score was improved from 64.00 to 90.32 points, and the authors indicated that remnant- 
preservation was unnecessary in ATFL reconstruction [29]. Jiang et al. conducted the non-remnantpreserving ATFL reconstruction to treat 25 CLAl cases, and satisfactory clinical effects were obtained after 3 years of follow-up [30]. The above results demonstrate that, favorable effects can be achieved in ATFL reconstruction, regardless of whether the ATFL remnant is preserved or not. To the best of our knowledge, there is no answer from existing literature about how to select. From the results of our 53 cases, the follow-up results at an average of 2.5 years after surgery in remnant-preserving group $(n=25)$ and non-remnant-preserving group $(n=28)$ revealed no statistical differences in VAS, AOFAS, KAFS, ATT, and AJPS between two groups. Therefore, we suggest that preserving the ATFL remnant in ATFL reconstruction displays no obvious short-term clinical significance.

This study has the following advantages: (1) it was a prospective randomized controlled study; (2) it assessed the postoperative ankle function, stability and proprioception; (3) it had a large sample size. Similarly, several limitations should also be noted in this study: (1) the measuring method for proprioceptive function was simple, which might not comprehensively assess the proprioception; (2) the follow-up time was short, while the research results might change with the increases in sample size and follow-up time; and (3) there was difference between clinical significance and statistical significance.

\section{Conclusions}

Compared with non-remnant-preserving ATFL reconstruction, the remnant-preserving ATFL reconstruction with autologous semitendinosus tendon as the graft shows no short-term advantages in clinical outcomes, such as postoperative ankle function, stability and proprioceptive recovery.

\section{Declarations}

\section{Acknowledgements}

Not applicable.

\section{Funding}

This study was supported by grants from the Jiangsu Provincial Medical Youth Talent Program (QNRC2016393) and Six Talent Peaks Project in Jiangsu Province (2019-WSW-173).

\section{Availability of data and materials}

All data generated or analyzed during this study are included in this published article. Shi-Ming Feng and Ai-Guo Wang can be contacted to request the raw data.

\section{Authors' contributions}

QQS performed the follow-up experiments. SMF gave the experiment guidance during this study and revised this paper critically for important intellectual content. NM and OF advised on the theoretical 
framework of the study, critically revising the procedures and the writing of the manuscript. AGW analyzed and interpreted the data and was a major contributor in writing the manuscript. All authors read and approved the final manuscript.

\section{Ethics approval and consent to participate}

The study was approved by the clinical research ethics committee of the Xuzhou Central Hospital. Reference No. ZXYY-2015090. Written informed consent was obtained by all participants.

\section{Consent for publication}

Not applicable.

\section{Competing interests}

The authors declare that they have no competing interests.

\section{Abbreviations}

ACL: Anterior cruciate ligament; AJPS: Active joint position sense; AOFAS: American Orthopedic Foot and Ankle Society; ATFL: Anterior talofibular ligament; ATT: Anterior talar translation; CLAl: Chronic lateral ankle instability; KAFS: Karlsson Ankle Functional Score; VAS: Visual Analogue Scale.

\section{References}

1. Azuma N, Sugano T, Shimizu I, Kosaka M. Injuries associated with Japanese high-school men's volleyball: a two-year survey and analysis. J Phys Ther Sci. 2019; 31:656-660.

2. Longo UG, Loppini M, Romeo G, van Dijk CN, Maffulli N, Denaro V. Bone bruises associated with acute ankle ligament injury: do they need treatment? Knee Surg Sports Traumatol Arthrosc. 2013; 21:1261-1268.

3. Lynch SA, Renström PA. Treatment of acute lateral ankle ligament rupture in the athlete. Conservative versus surgical treatment. Sports Med.1999; 27:61-71.

4. Samoto N, Sugimoto K, Takaoka T, Fujita T, Kitada C, Takakura Y. Comparative results of conservative treatments for isolated anterior talofibular ligament (ATFL) injury and injury to both the ATFL and calcaneofibular ligament of the ankle as assessed by subtalar arthrography. $\mathrm{J}$ Orthop Sci.2007; 12:49-54.

5. Maffulli N, Del Buono A, Maffulli GD, et al. Isolated anterior talofibular ligament Broström repair for chronic lateral ankle instability: 9-year follow-up. Am J Sports Med. 2013; 41:858-864

6. Cordier G, Ovigue J, Dalmau-Pastor M, Michels F. Endoscopic anatomic ligament reconstruction is a reliable option to treat chronic lateral ankle instability. Knee Surg Sports Traumatol Arthrosc. 2019; doi: 10.1007/s00167-019-05793-9. 
7. Ferran NA, Oliva F, Maffulli N. Ankle instability. Sports Med Arthrosc Rev. 2009; 17:139-145.

8. Lee BI, Kim BM, Kho DH, Kwon SW, Kim HJ, Hwang HR. Does the tibial remnant of the anterior cruciate ligament promote ligamentization? Knee. 2016; 23:1133-1142.

9. Haraguchi N, Tokumo A, Okamura R, et al. Influence of activity level on the outcome of treatment of lateral ankle ligament rupture. J Orthop Sci. 2009; 14: 391-396.

10. Beynnon BD, Webb G, Huber BM, Pappas CN, Renström P, Haugh LD. Radiographic measurement of anterior talar translation in the ankle: determination of the most reliable method. Clin Biomech (Bristol, Avon). 2005; 20:301-306.

11. Bae YS. Effects of spiral taping on proprioception in subjects with unilateral functional ankle instability. J Phys Ther Sci. 2017; 29:106-108.

12. Camacho LD, Roward ZT, Deng Y, Latt LD. Surgical Management of Lateral Ankle Instability in Athletes. J Athl Train. 2019; $54: 639-649$.

13. Song Y, Li H, Sun C, et al. Clinical Guidelines for the Surgical Management of Chronic Lateral Ankle Instability: A Consensus Reached by Systematic Review of the Available Data. Orthop J Sports Med. 2019; 7:2325967119873852.

14. Diniz P, Pacheco J, Flora M, et al. Clinical applications of allografts in foot and ankle surgery. Knee Surg Sports Traumatol Arthrosc. 2019; 27:1847-1872.

15. Porter DA, Kamman KA. Chronic Lateral Ankle Instability: Open Surgical Management. Foot Ankle Clin. 2018; 23:539-554.

16. Maffulli N, Ferran NA. Management of acute and chronic ankle instability. J Am Acad Orthop Surg. 2008; 16:608-615.

17. Halasi T, Kynsburg A, Tállay A, Berkes I. Changes in joint position sense after surgically treated chronic lateral ankle instability. Br J Sports Med. 2005; 39:818-824.

18. Urgüden M, Kızılay F, Sekban H, Samancı N, Ozkaynak S, Ozdemir H. Evaluation of the lateral instability of the ankle by inversion simulation device and assessment of the rehabilitation program. Acta Orthop Traumatol Turc. 2010; 44:365-77.

19. Brown AJ, Shimozono Y, Hurley ET, Kennedy JG. Arthroscopic versus open repair of lateral ankle ligament for chronic lateral ankle instability: a meta-analysis. Knee Surg Sports Traumatol Arthrosc. 2018; doi: 10.1007/s00167-018-5100-6.

20. Yasui Y, Shimozono Y, Kennedy JG. Surgical Procedures for Chronic Lateral Ankle Instability. J Am Acad Orthop Surg. 2018; 26:223-230.

21. Guelfi M, Zamperetti M, Pantalone A, Usuelli FG, Salini V, Oliva XM. Open and arthroscopic lateral ligament repair for treatment of chronic ankle instability: A systematic review. Foot Ankle Surg. 2018; 24:11-18.

22. Yeo ED, Lee KT, Sung IH, Lee SG, Lee YK. Comparison of All-Inside Arthroscopic and Open Techniques for the Modified Broström Procedure for Ankle Instability. Foot Ankle Int. 2016; 37:1037-1045. 
23. Teixeira J, Guillo S. Arthroscopic Treatment of Ankle Instability - Allograft/Autograft Reconstruction. Foot Ankle Clin. 2018; 23:571-579.

24. Dierckman BD, Ferkel RD. Anatomic Reconstruction With a Semitendinosus Allograft for Chronic Lateral Ankle Instability. Am J Sports Med. 2015; 43:1941-1950.

25. de Vasconcelos GS, Cini A, Sbruzzi G, Lima CS. Effects of proprioceptive training on the incidence of ankle sprain in athletes: systematic review and meta-analysis. Clin Rehabil. 2018; 32:1581-1590.

26. McCriskin BJ, Cameron KL, Orr JD, Waterman BR. Management and prevention of acute and chronic lateral ankle instability in athletic patient populations. World J Orthop. 2015; 6:161-171.

27. Wang HD, Wang FS, Gao SJ, Zhang YZ. Remnant preservation technique versus standard technique for anterior cruciate ligament reconstruction: a meta-analysis of randomized controlled trials. $J$ Orthop Surg Res. 2018; 13:231.

28. Dong P, Gu S, Jiang Y, et al. All arthroscopic remnant-preserving reconstruction of the lateral ligaments of the ankle: A biomechanical study and clinical application. Biochem Biophys Res Commun. 2018; 505:985-990.

29. Wang W, Xu GH. Allograft tendon reconstruction of the anterior talofibular ligament and calcaneofibular Ligament in the treatment of chronic ankle instability. BMC Musculoskelet Disord. 2017; 18:150.

30. Matheny LM, Johnson NS, Liechti DJ, Clanton TO. Activity Level and Function After Lateral Ankle Ligament Repair Versus Reconstruction. Am J Sports Med. 2016; 44:1301-1308.

\section{Tables}

Table 1 Characterization of the Sample

\begin{tabular}{lccc}
\hline Variable & Preservation Group $(\mathrm{n}=25)$ & Non-Preservation Group $(\mathrm{n}=28)$ & $P^{*}$ Value \\
\hline Age, $\mathrm{y}$ & $34.18 \pm 7.63$ & $32.85 \pm 9.07$ & $0.565 \ddagger$ \\
Sex & & & $0.805 \dagger$ \\
Male & 18 & 21 & \\
Female & 7 & 7 & \\
BMI, kg $/ \mathrm{m}^{2}$ & $23.18 \pm 3.02$ & $22.75 \pm 2.36$ & $0.570 \ddagger$ \\
VAS & $1.22 \pm 0.86$ & $1.14 \pm 0.92$ & $0.745 \ddagger$ \\
AOFAS & $52.19 \pm 8.20$ & $54.33 \pm 7.27$ & $0.322 \ddagger$ \\
KAFS & $58.21 \pm 10.05$ & $60.14 \pm 9.43$ & $0.476 \ddagger$ \\
ATT, mm & $7.96 \pm 2.12$ & $7.87 \pm 2.35$ & $0.884 \ddagger$ \\
Disease duration, mo & $13.27 \pm 4.96$ & $14.14 \pm 5.28$ & $0.539 \ddagger$ \\
\hline
\end{tabular}


Abbreviations: BMI: Body Mass Index; STS, Sinus Tarsi Syndrome; VAS, Visual analogue scale; AOFAS, American Orthopaedic Foot and Ankle Society; KAFS, Karlsson Ankle Functional Score; ATT, Anterior Talar Translation.

*A value $\mathrm{p}<0.05$ was set as statistically significant.

†Pearson $\chi^{2}$ test.

抽 test.

Table 2 Comparison of the Two Groups

\begin{tabular}{lccc}
\hline Variable & Preservation Group (n=25) & Non-Preservation Group (n=28) & $P^{*}$ Value \\
\hline Operative time, min & $71.63 \pm 5.75$ & $70.18 \pm 6.12$ & $0.378 \dagger$ \\
Hospital stay & & & \\
$\quad$ duration, days & $3.12 \pm 1.31$ & $3.14 \pm 1.28$ & $0.956 \dagger$ \\
VAS & $7.43 \pm 2.17$ & $7.16 \pm 3.05$ & $0.710 \dagger$ \\
AOFAS & $89.20 \pm 5.14$ & $87.77 \pm 4.36$ & $0.283 \dagger$ \\
KAFS & $88.01 \pm 4.72$ & $87.15 \pm 4.58$ & $0.505 \dagger$ \\
ATT, mm & $2.87 \pm 0.91$ & $2.90 \pm 0.89$ & $0.904 \dagger$ \\
AJPS, degree & & & \\
Inversion 10 & $8.03 \pm 1.26$ & $7.87 \pm 1.19$ & $0.638 \dagger$ \\
Inversion 20 & $18.62 \pm 1.75$ & $18.33 \pm 2.01$ & $0.577 \dagger$ \\
Plantar flexion $10^{\circ}$ & $7.52 \pm 1.46$ & $7.39 \pm 1.92$ & $0.781 \dagger$ \\
Plantar flexion $20^{\circ}$ & $19.06 \pm 2.13$ & $18.71 \pm 1.88$ & $0.531 \dagger$ \\
Follow-up time, mo & $30.35 \pm 9.82$ & $31.41 \pm 9.25$ & $0.689 \dagger$ \\
\hline
\end{tabular}

Abbreviations: VAS, Visual analogue scale; AOFAS, American Orthopaedic Foot and Ankle Society; KAFS, Karlsson Ankle Functional Score; ATT, Anterior Talar Translation; AJPS, Active Joint Position Sense.

$*_{A}$ A value $p<0.05$ was set as statistically significant.

$\dagger \mathrm{t}$ test.

\section{Figures}



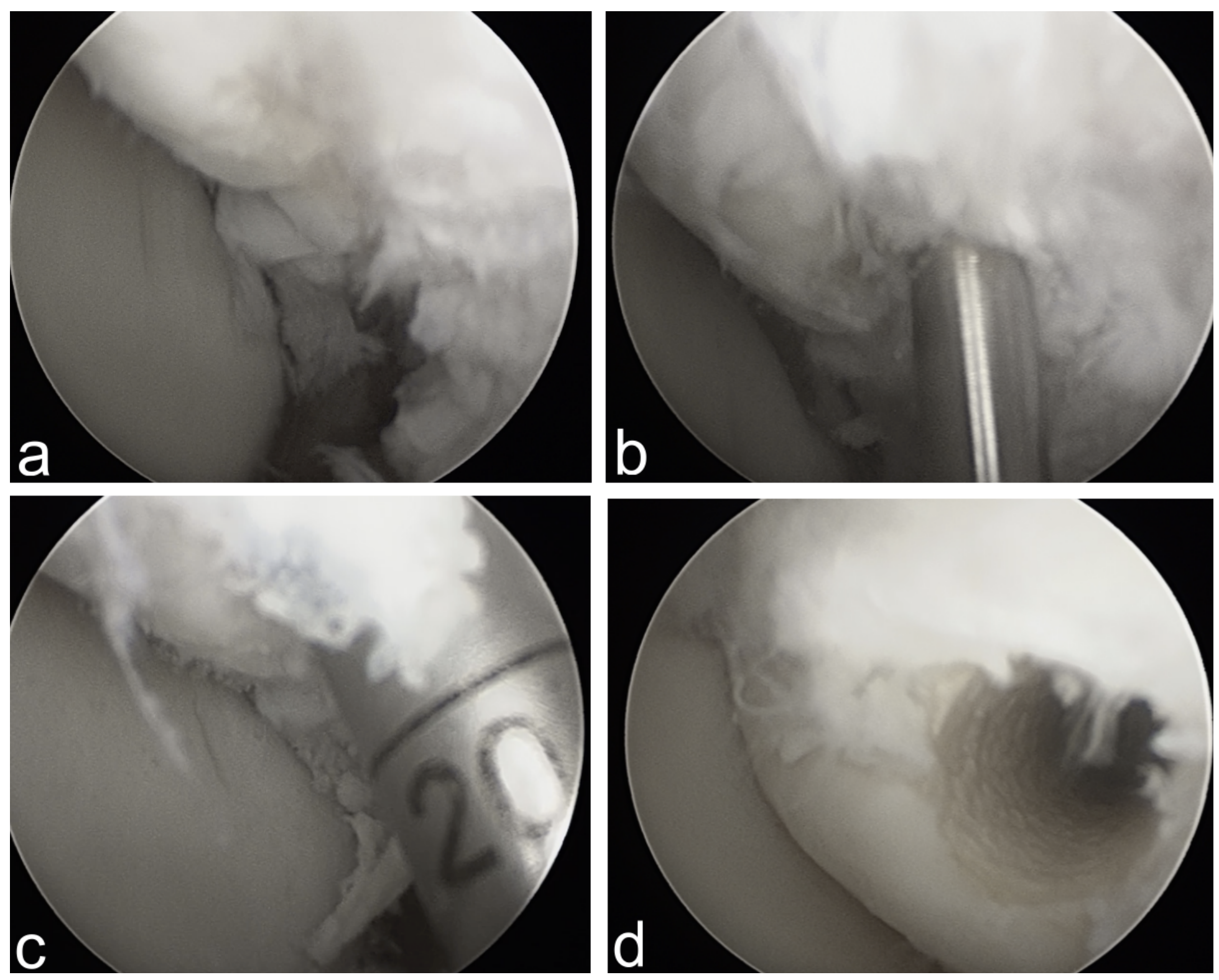

Figure 1

ATFL footprint and fibular tunnel. a Evaluation of the quality of ATFL under the arthroscopy, and the fibular remnant of ATFL was preserved. b A guidewire was inserted into a central site closely adjacent to the fibular remnant of ATFL. $c$ An electric drill bit following the guidewire was used to make a fibular tunnel. $d$ The fibular tunnel was made closely adjacent to the remnant of ATFL.
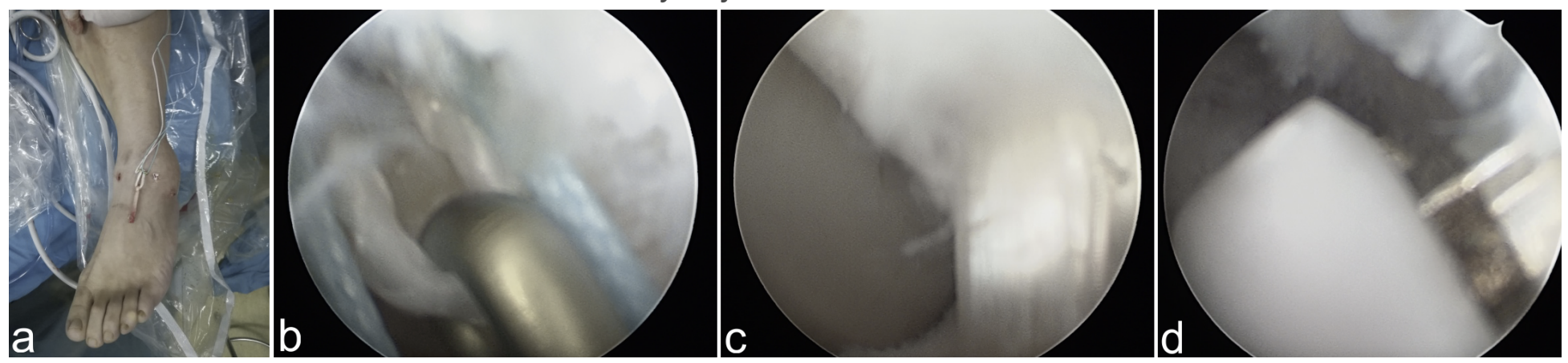

Figure 2 
Semitendinosus tendon translation and fixation. a The semitendinosus tendon was penetrated into the button plate (Smith \& Nephew, USA) at equivalent length, and the both sides of tendon were sutured. b A guide pin with a wire was used to penetrate the button plate into the fibular tunnel. $c$ the other terminal of tendon graft was pulled back to better confirm the button plate fixing effect and the tendon condition of the remnant site. $d$ After the grafted tendon was strained, and a $6.0 \mathrm{~mm}$ interface screw (Smith \& Nephew, USA) was used to fix the distal end of grafted tendon inside the talar tunnel.
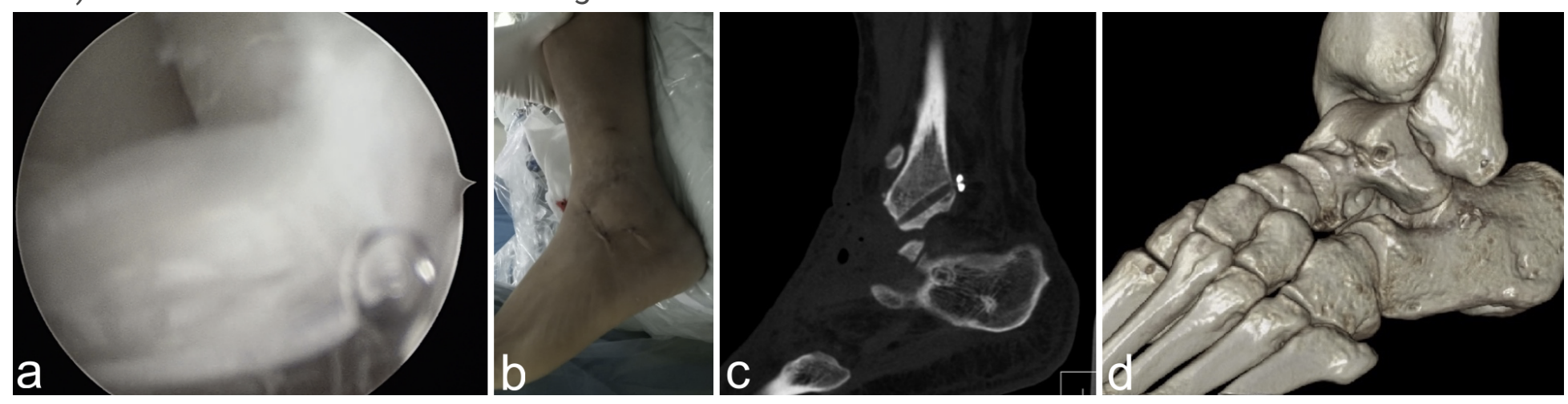

\section{Figure 3}

Final result with reconstructed ATFL. a Evaluation of the reconstructed ATFL. b The appearance of surgical portals after the operation. c Postoperative CT scan shows the fibular tunnel and the button plate of the reconstructed ATFL. d Postoperative 3D CT scan shows the bony insertion sites of the talus and fibular. 